\title{
Haplotype-assisted accurate non-invasive fetal whole genome recovery through maternal plasma sequencing
}

Shengpei Chen ${ }^{1,2+}$, Huijuan Ge ${ }^{1 \dagger}$, Xuebin Wang ${ }^{1 \dagger}$, Xiaoyu Pan ${ }^{1,3 \dagger}$, Xiaotian Yao ${ }^{1}$, Xuchao Li ${ }^{1}$, Chunlei Zhang ${ }^{1}$, Fang Chen ${ }^{1}$, Fuman Jiang ${ }^{1}$, Peipei Li ${ }^{1}$, Hui Jiang ${ }^{1}$, Hancheng Zheng ${ }^{1}$, Lei Zhang ${ }^{1}$, Lijian Zhao', Wei Wang ${ }^{1}$, Songgang $\mathrm{Li}^{1}$, Jun Wang ${ }^{1}$, Jian Wang ${ }^{1}$, Huanming Yang ${ }^{1}$, Yingrui $\mathrm{Li}^{1^{*}}$ and Xiuqing Zhang ${ }^{{ }^{*}}$

See related Commentary article: http://www.biomedcentral.com/1741-7015/11/56

\begin{abstract}
Background: The applications of massively parallel sequencing technology to fetal cell-free DNA (cff-DNA) have brought new insight to non-invasive prenatal diagnosis. However, most previous research based on maternal plasma sequencing has been restricted to fetal aneuploidies. To detect specific parentally inherited mutations, invasive approaches to obtain fetal DNA are the current standard in the clinic because of the experimental complexity and resource consumption of previously reported non-invasive approaches.

Methods: Here, we present a simple and effective non-invasive method for accurate fetal genome recoveryassisted with parental haplotypes. The parental haplotype were firstly inferred using a combination strategy of trio and unrelated individuals. Assisted with the parental haplotype, we then employed a hidden Markov model to non-invasively recover the fetal genome through maternal plasma sequencing.

Results: Using a sequence depth of approximately 44X against a an approximate 5.69\% cff-DNA concentration, we non-invasively inferred fetal genotype and haplotype under different situations of parental heterozygosity. Our data show that $98.57 \%, 95.37 \%$, and $98.45 \%$ of paternal autosome alleles, maternal autosome alleles, and maternal chromosome $\mathrm{X}$ in the fetal haplotypes, respectively, were recovered accurately. Additionally, we obtained efficient coverage or strong linkage of $96.65 \%$ of reported Mendelian-disorder genes and $98.90 \%$ of complex diseaseassociated markers.
\end{abstract}

Conclusions: Our method provides a useful strategy for non-invasive whole fetal genome recovery.

\section{Background}

Prenatal diagnosis is one of the most efficient approaches to decrease the incidence of birth defects [1]. Traditionally, fetal cells for prenatal diagnosis are collected invasively by the procedures of amniocentesis or chorionic villus sampling (CVS), but these carry a risk of miscarriage $[2,3]$. To reduce the requirement of invasive testing, non-invasive approaches, such as the use of maternal serum markers and ultrasound, are widely used in the clinic to classify low- and high-risk pregnant women with Down's

\footnotetext{
*Correspondence: liyr@genomics.cn; zhangxq@genomics.cn

† Contributed equally

${ }^{1}$ BGI-Shenzhen, Shenzhen 518083, China

Full list of author information is available at the end of the article
}

syndrome fetuses. However, these non-invasive prenatal screens are unsatisfactory to many clinicians and pregnant women due to their false-positive and potential false-negative rates [4-6]. With the discovery of cell-free fetal DNA (cff-DNA) in maternal plasma [7-10] and the emergence of high-throughput sequencing, the clinical application of non-invasive tests to detect fetal chromosomal abnormalities using maternal plasma sequencing have been discussed [11-13].

Theoretically, it should be possible to recover the fetal genome non-invasively through maternal plasma sequencing to enable the comprehensive prenatal diagnosis of Mendelian diseases and lessen the need for invasive procedures $[14,15]$. In 2010, Lo's group showed the feasibility of 
non-invasive fetal whole genome recovery and inferring the fetal genotype, although they did not assess the biparentally heterozygous sites in the fetal genome [14]. Recent studies from Kitzman et al. [15] and Fan et al. [16] introduced accurate non-invasive fetal genotype inference methods assisted by maternal haplotype, but their methods showed uncertain performance in detecting paternal transition in low cff-DNA concentrations. In early gestation, the concentration of cff-DNA is approximately $3 \%$ to $6 \%$ of the total cell-free DNA [17], which may lead to uneven recovery of the paternal allele in the whole genome. Robust strategies of noninvasively detecting both maternal and paternal alleles are still needed. Moreover, the fetal haplotype information is especially useful in detecting some haplotype-related diseases, such as systemic lupus erythematosus [18], as well as personal genomic analyses in the future $[19,20]$.

Here, we developed a novel strategy of fetal genome recovery, inferring the fetal genotype as well as haplotype at the same time. Given the fact that the fetal genome is the combination of parentally transmitted chromosomes, we reconstructed the fetal genome by observing parental allele transition in maternal plasma. We first used a combined strategy of trios and unrelated individuals to construct parental haplotypes, and then observed the parental allele transition in maternal plasma and optimized the fetal haplotype using a hidden Markov model (HMM) and Viterbi algorithm. Thereby, we recovered the fetal haplotype as well as the genotype against all parental heterozygosity in one step. Our method highlights the prospective value to translational medicine of non-invasive prenatal diagnosis to recover the fetal genome using maternal plasma sequencing.

\section{Methods}

\section{Sample preparation}

In this study, a Chinese couple and both parents of the couple were recruited with written informed consent. Also, this study was approved by the institutional review board of BGI-Shenzhen and conducted in accordance with the Declaration of Helsinki.

\section{Peripheral blood}

We collected $10 \mathrm{~mL}$ of peripheral blood from a woman with pregnancy of 13 weeks of gestation, $5 \mathrm{~mL}$ of peripheral blood from her husband, and $10 \mathrm{~mL}$ of fetal umbilical blood after the delivery. Blood samples from each participant were collected in EDTA-containing tubes.

\section{Maternal plasma}

We obtained maternal plasma from $10 \mathrm{~mL}$ maternal peripheral blood after centrifugation at $1,600 \mathrm{~g}$ for $10 \mathrm{~min}$. Great care was taken in the collection of plasma samples to avoid taking the buffy coat or any blood clots. Plasma was transferred to $2.0 \mathrm{~mL}$ eppendorf tubes and centrifuged at $16,000 \mathrm{~g}$ for $10 \mathrm{~min}$ to remove residual cells. Blood samples and plasma samples were stored at $-20^{\circ} \mathrm{C}$ and $-80^{\circ} \mathrm{C}$, respectively, until further processing.

\section{Saliva}

Saliva was collected from grandparents using Oragene ${ }^{\circledR}$ OG-250 tubes and kits, following the standard manufacturer's instructions.

\section{DNA extraction}

g-DNA from whole blood, saliva, and maternal plasma were extracted by using a TIANamp Micro DNA Kit (Tiangen) according to the manufacturer's instructions.

\section{Library preparation and massively parallel genomic sequencing \\ Genomic DNA}

One microgram of g-DNA was sheared by an S2 sonicator (Covaris, Inc.), yielding fragments between 100 and $500 \mathrm{bp}$, with a predominance of $300 \mathrm{bp}$. For massively parallel genomic sequencing, approximately $1 \mu \mathrm{g}$ of fragmented g-DNA was prepared for library construction. Briefly, DNA fragments were blunt-ended using T4 DNA polymerase (Enzymatics), Klenow polymerase (Enzymatics), and T4 polynucleotide kinase (Enzymatics) and were ligated to adapters after addition of terminal A nucleotides. The adapter-ligated DNA fragments in the range of 300 to $350 \mathrm{bp}$ were size-selected using $2 \%$ agarose electrophoresis and then amplified using a 10-cycle PCR. An Agencourt AMPure $450 \mathrm{~mL}$ Kit was used for the purification of PCR products.

\section{Plasma DNA}

Plasma DNA (10 to $50 \mathrm{ng}$ ) was used for library preparation according to a modified protocol, in which a 17cycle PCR was conducted to enrich adapter-ligated DNA fragments.

\section{Library QC and sequencing}

The libraries were quality-controlled by using an Agilent DNA 1000 kit on the 2100 Bioanalyzer (Agilent) platform and quantified by real-time PCR. DNA libraries were hybridized to the surface of sequencing flowcells, and DNA clusters were generated after amplification. The libraries were then sequenced using the Illumina Hiseq $^{\mathrm{TM}} 2000$ sequencing system according to the manufacturer's instructions. The sequence reads of this parent-offspring trio have been uploaded to the NCBI SRA database (SRA060043).

\section{Illumina DNA microarray}

The construction of the library and scanning of the microarray (Omni 2.5 SNP-array) were done according to the manufacturer's instructions for the corresponding array and for Iscan. 


\section{Bioinformatics}

Bioinformatic analyses are described in the Additional file 1 (Additional file 1, Supplementary Methods).

\section{Results}

Accurate fetal genome recovery through maternal plasma

To perform haplotype-assisted accurate non-invasive fetal whole genome recovery through maternal plasma sequencing (Figure 1), we recruited a Chinese woman with pregnancy of 13 weeks of gestation and her family, including three generations, as well as fetal blood after delivery. We then performed approximately $44 \mathrm{X}$ and 20X whole genome shotgun sequencing of the plasma sample and of parental genomic DNA (g-DNA), respectively (Table 1). The cff-DNA concentration of this male fetus was estimated as $5.69 \%$ using the biparentally homozygous sites. Illumina Infinium HD Human610Quad BeadChip was used to genotype the gDNA from grandparents to construct the parental haplotypes. Also, we used Illumina HumanOmni2.5-8 BeadChip to validate the accuracy of parental SNP calling, in which the parental genotypes were validated as approximately 99.22\% consistent with the array (Table 1 and Additional file 1, Table S1).

We then performed a parental haplotype construction with a combined strategy of trios and unrelated individuals. Although both trios and unrelated individuals could be applied to construct the parental haplotypes, the haplotype ambiguity in trio strategy and the stratification in unrelated individual strategy would significantly restrict the value of either of these strategies. In this study, the parental haplotypes were obtained by BEAGLE [21] using their sequencing genotype and the genotyping data of the grandparents along with the newly released 51 parent-offspring trios of Chinese Han in the 1000 Genomes project (pilot II). By using this strategy, the inferred rate of parental haplotypes increased, on average, from $90.32 \%$ to $100 \%$ compared to using a trio strategy only.

Assisted by the parental haplotypes, we then developed an efficient method for fetal whole genome recovery through maternal plasma sequencing. Ideally, in maternal plasma sequencing with a site-by-site strategy (SBSS), we could reconstruct the paternally transmitted allele directly by determining the nucleotide sequence of the paternal-specific allele at paternal-only heterozygous sites and determine the maternal transition by observing allelic imbalance at individual sites. However, the application of this simple idea could be hinderedby low cffDNA concentration and sequence depth. In our plasma sequencing, approximately $57.84 \%$ of the paternal-specific alleles were totally absent (Figure S3). Additionally, our estimation of the concentrations of three different alleles in plasma showed that 24,938 of 137,567 (25.40\%) sites showed an opposite allelic imbalance (Additional file 1, Supplementary Materials). These results indicate the infeasibility of SBSS in samples with low cff-DNA concentration and sequence depth. Thus, we introduced a sensitive HMM to identify the parentally transmitted allele and recombination breakpoints (Figure 2 and Additional file 1, Supplementary Methods), in which we predicted the fetal haplotype on the paternal-only, maternal-only, and biparentally heterozygous sites in one step. With the use of the HMM and Viterbi algorithm, the fetal haplotypes of the 374,980 markers (including chromosome $\mathrm{X}$ ) were recovered successfully (Table 2).

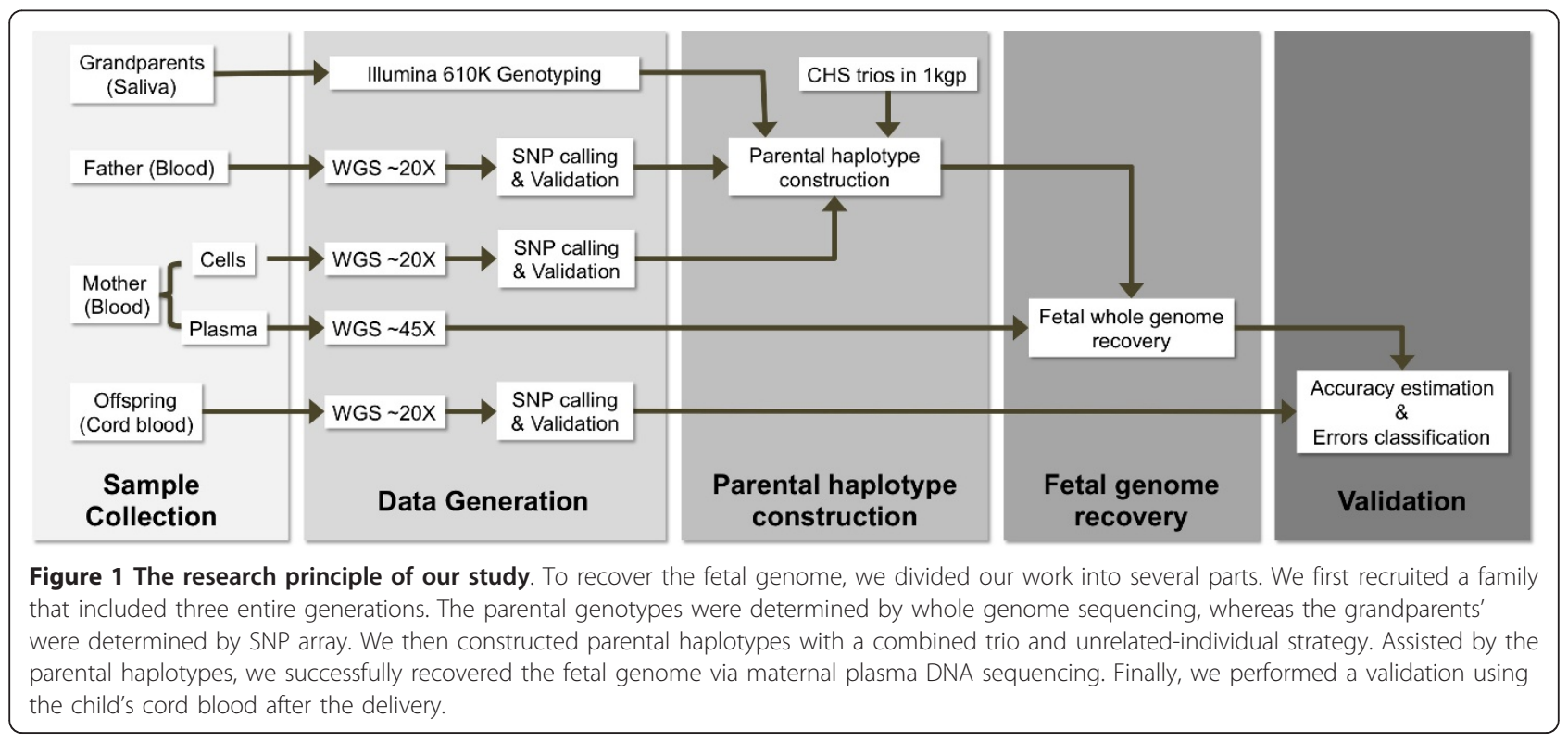


Table 1 Data production

\begin{tabular}{|c|c|c|c|c|c|c|c|}
\hline \multicolumn{8}{|c|}{ Microarray array } \\
\hline Samples & Type of DNA & \multicolumn{2}{|c|}{ Type of microarray } & \multicolumn{2}{|l|}{ Call rate (\%) } & \multicolumn{2}{|c|}{ SNP calling $(n)\left(10^{5}\right)$} \\
\hline Grandparents $^{a}$ & g-DNA (saliva) & \multicolumn{2}{|c|}{ Human 610-Quad BeadChip } & \multicolumn{2}{|l|}{$99.70 \pm 0.07$} & \multicolumn{2}{|c|}{$5.89 \pm 0.004$} \\
\hline \multicolumn{8}{|l|}{ WGS } \\
\hline Samples & Type of DNA & $\begin{array}{l}\text { Reads }(n) \\
\left(10^{9}\right)\end{array}$ & $\begin{array}{l}\text { Production } \\
\text { (Gb) }\end{array}$ & $\begin{array}{l}\text { Map rate } \\
(\%)\end{array}$ & $\begin{array}{l}\text { Coverage } \\
(\%)\end{array}$ & $\begin{array}{l}\text { Depth } \\
\text { (fold) }\end{array}$ & $\begin{array}{l}\text { Consistency in validation } \\
(\%)\end{array}$ \\
\hline Father & g-DNA (blood) & 0.72 & 71.89 & 89.75 & 99.71 & 21.86 & 99.23 \\
\hline Mother & g-DNA (blood) & 0.74 & 74.03 & 90.19 & 99.09 & 20.96 & 99.19 \\
\hline Offspring & $\begin{array}{l}\text { g-DNA (cord } \\
\text { blood) }\end{array}$ & 0.72 & 72.17 & 90.64 & 99.75 & 21.32 & 99.25 \\
\hline Plasma & Plasma DNA & 1.81 & 179.63 & 83.68 & 99.47 & 43.91 & - \\
\hline
\end{tabular}

${ }^{\mathrm{a}}$ Mean \pm standard deviation.

\section{Accuracy of the recovered fetal haplotype}

We performed a final validation to estimate the overall accuracy of the predicted fetal haplotype. To assess the standard fetal haplotype, we also performed a whole genome sequencing of the cord blood obtained after the child's birth to approximately 20 -fold coverage (Table 1 and Additional file 1, Table S1). The genotypes of the child were determined using SOAPsnp and were validated at $99.25 \%$ consistency with his genotyping of HumanOmni2.5-8 BeadChip. The standard haplotype of the child was inferred by the same method as used for his parents. Finally, the general accuracy of the paternal alleles and maternal alleles were estimated by comparing the recovered fetal genome with the standard haplotype

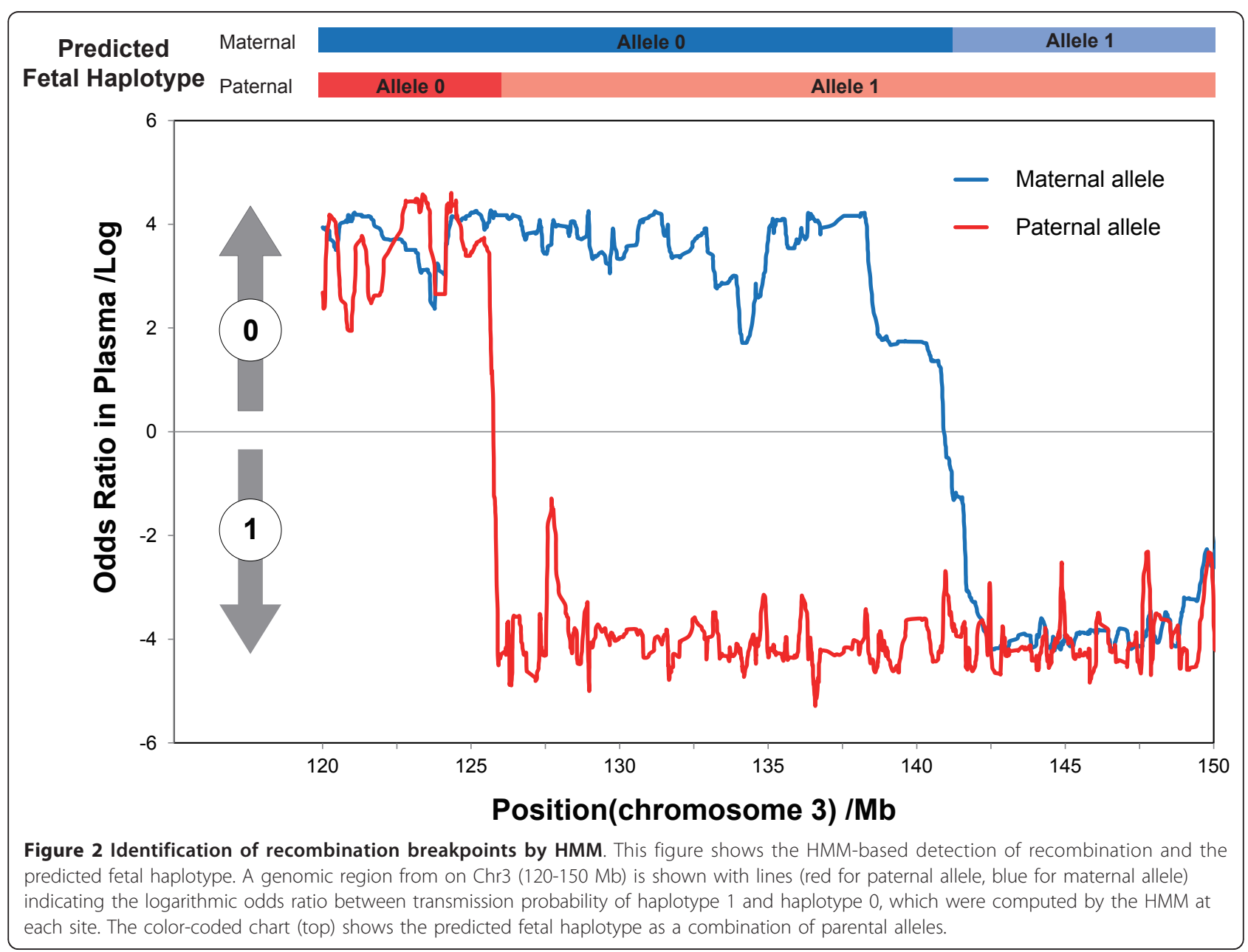


Table 2 The general accuracy of haplotype prediction

\begin{tabular}{|c|c|c|c|c|}
\hline \multirow[t]{2}{*}{ Category } & & \multirow{2}{*}{$\begin{array}{l}\text { Paternal allele } \\
\text { Autosome }\end{array}$} & \multicolumn{2}{|l|}{ Maternal allele } \\
\hline & & & Autosome & ChrX \\
\hline Consistent with g-DNA from cord blood & Loci ( $n$, percentage) & $\begin{array}{l}105,729 \\
(98.57 \%) \\
\end{array}$ & $\begin{array}{l}103,082 \\
(95.37 \%) \\
\end{array}$ & $\begin{array}{l}1,902 \\
(98.45 \%) \\
\end{array}$ \\
\hline \multirow{4}{*}{$\begin{array}{l}\text { Inconsistent with g-DNA from cord } \\
\text { blood }\end{array}$} & Loci ( $n$, percentage) & $1,529(1.43 \%)$ & $5,005(4.63 \%)$ & $30(1.55 \%)$ \\
\hline & Type I (noisy from haplotype inference) & $1,458(95.36 \%)$ & $1,442(28.81 \%)$ & - \\
\hline & Type II (recombination breakpoint related) & $71(4.64 \%)$ & $3,295(65.83 \%)$ & $24(80.00 \%)$ \\
\hline & $\begin{array}{l}\text { Type III (centromere or chromosome edge } \\
\text { related) }\end{array}$ & $0(0 \%)$ & $268(5.35 \%)$ & $6(20.00 \%)$ \\
\hline Total & & 107,258 & 108,087 & 1,932 \\
\hline
\end{tabular}

of the child (Table 2). For the recovered paternal alleles, 105,729 loci of our recovery were consistent with the standard haplotype, indicating a high accuracy of $98.57 \%$. For the recovered maternal autosomal alleles, 103,082 loci were consistent with the standard haplotype, for a slightly lower accuracy of maternal allele recovery of $95.37 \%$. The maternal allele recovery on the chromosome X showed an accuracy of $98.45 \%(1,902 /$ 1,932 loci).

We further classified the recovery errors into different types (Table 2). Type I errors, which were randomly distributed throughout the whole genome, explained $95.36 \%$ and $28.81 \%$ of paternal and maternal recovery inaccuracies, respectively. We assume that type I errors were caused by the haplotype ambiguity during the parental or standard fetal haplotype inference. The type II errors, which mostly clustered next to the recovered recombination breakpoints, were most probably caused by the inaccuracy of recombination breakpoint recovery. This type of error explained the remaining $4.64 \%$ of the paternal allele recovery inaccuracies, $65.83 \%$ of the maternal autosome allele recovery mistakes, and $80.00 \%$ of chromosome $\mathrm{X}$ recovery mistakes, indicating difficulties in maternal recombination breakpoint determination. The rest, referred to as type III errors, were related to heterochromatin close to the centromeres or chromosome ends. Type III errors explained $5.35 \%$ of maternal autosome and $20.00 \%$ of chromosome X maternal allele recovery errors (Additional file 1, Table S5).

To estimate the correlation between sequencing data and the detection accuracy of the recovered fetal genome, we sampled a subset of data from the maternal plasma sequencing (Figure 3). Generally, the accuracy of the recovered fetal genome increased with the depth of maternal plasma sequencing. Because of the existence of type I errors, the accuracy began to stabilize when the sequence depth grew $>20 X$. Additionally, the accuracy of the paternal-only heterozygous sites indicated the robustness of our method for paternal allele recovery in low cffDNA concentrations among different sequence depths.
For example, using only $6 \%$ of the plasma sequence data (non-duplicate approximately 2.01X), we successfully recovered $97.61 \%$ of the maternal-only heterozygous sites.

In summary, $98.57 \%$ of the paternal alleles, $95.37 \%$ of the maternal autosomes and $98.45 \%$ of the chromosome $\mathrm{X}$ were recovered precisely using approximately $43.91 \mathrm{X}$ (non-duplicate approximately 33.60X) maternal plasma sequencing with a $5.69 \%$ cff-DNA concentration. The quality of haplotype interference, the accuracy of the recombination breakpoint prediction, and heterochromatin close to centromeres and chromosome ends explained most of our recovery errors. The simulations suggested the robustness of our method at lower sequence depth and low cff-DNA concentration, especially for paternal allele recovery.

\section{The application of non-invasive fetal genomics}

Inheritable genetic disease screening and Mendelian character predictions are two important applications of accurate fetal genome recovery. So far, 7,895 pathogenic genes related to Mendelian diseases have been released by OMIM (Online Mendelian Inheritance in Man [22]), $96.65 \%$ of which were directly covered by or strongly linked with our 374,980 effective marker loci. In the case of complex diseases, $98.90 \%$ of 6,939 disease-associated loci from the NHGRI GWAS Catalog [23] were directly covered by or strongly linked with our marker loci. Interestingly, a TC genotype at rs17822931 (Chr16: $46,815,699$; predicted accurately) is consistent with the offspring having earwax of the wet type [24], which is not typical in Asians and Native Americans [25]. The level of throughput of disease and trait screening implies that noninvasive prenatal diagnosis/screening can have high detection efficiency. The strategy of using three generations of a family increased the robustness of detecting rare mutations, showing a similar performance between common mutations and rare mutations (Additional file 1, Figure S4). Moreover, based on our accurate recovered haplotype, heritable complex variations 


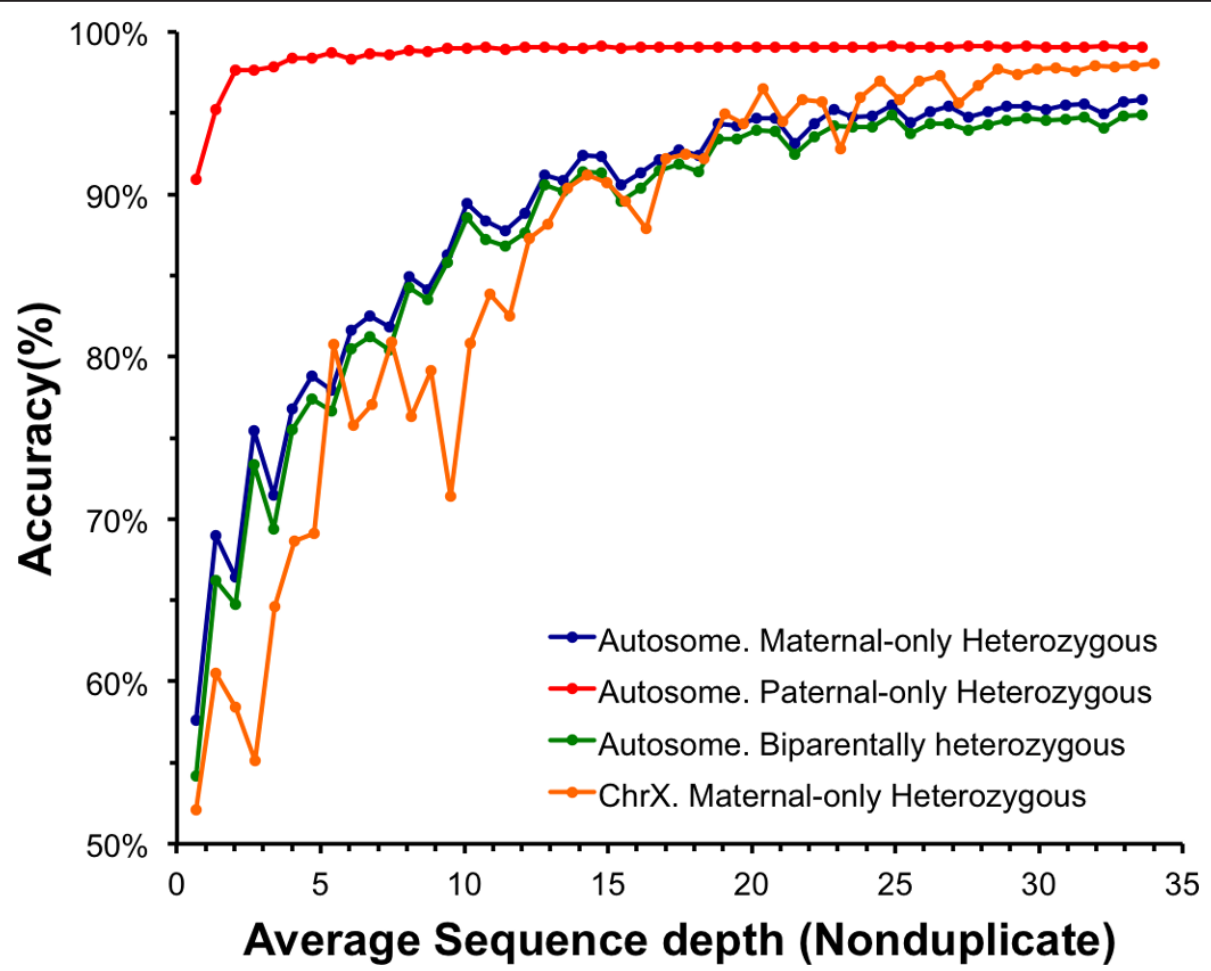

Figure 3 The relationship between accuracy and sequence depth. The color-coded curves denote statistics at different kinds of sites (blue: autosome, maternal-only heterozygous sites; red: autosome, paternal-only heterozygous sites; green: autosome, biparentally heterozygous sites; orange: ChrX, maternal heterozygous sites).

(such as long insertions/deletions, translocations, rearrangements, and even disease-related methylation), unlike SNPs, which were hard to observe directly in maternal plasma, could be mapped to the fetal genome by their linkage disequilibrium relationships using other existing techniques.

\section{Discussion}

Here we report a haplotype-assisted approach for noninvasive fetal whole genome recovery. The characteristics of our method and two previously reported haplotypeassisted methods $[15,16]$ are summarized in Table 3 . We further assessed the fetal genotype accuracy to compare the practical performance in corresponding cases to reach comprehensive conclusions (Table 4).

Three fetal genome recovery strategies have employed parental haplotypes, but with different inference strategies. We used a common genetics approach to determine the parental haplotypes by using the genotyping data of surviving grandparents or born offspring. This approach provided a practical strategy for non-invasive fetal genome screening for families with probands, especially for families with born offspring with Mendelian diseases. However, this specific sample recruitment would restrict its prospects for clinical application. To overcome this limitation, Kitzman et al. [26] and Fan et al. [27] have constructed maternal haplotypes directly using noninvasive experimental approaches. However, the time and resource consumption of their complex experimental methods would restrict their clinical application. For example, it would take approximately 8 days and another US\$3,678 to prepare the fosmid clone library [26].

Second, these three strategies all used the maternal haplotype, but we used the paternal haplotype. For paternal-only heterozygous sites, Kitzman et al. performed SBSS to detect the paternal-specific allele. In SBSS, one or more reads matching the paternal-specific allele are taken as evidence of its transition. However, the performance of SBSS depends on cff-DNA concentration and sequence depth. For example, $96.80 \%$ of alleles in the trio labeled I1 in the Kitzman et al. study (WGS approximately 78X, cff-DNA concentration approximately 13\%) were predicted correctly. However, in the case of low cffDNA concentration, such as trio G1 (WGS approximately 56X, at 8.14 weeks) reported by Kitzman et al., only $60.3 \%$ of paternal-specific alleles were identified correctly. Therefore, Fan et al. imputed the paternal allele using data from the 1000 Genomes project. In total, approximately $70 \%$ of the paternally transmitted alleles were reconstructed with an accuracy of $93 \%$ to $97 \%$. This implied the imperfect efficiency and eurytopicity of SBSS, even with population-scale sequencing for imputation. 
Table 3 Comparison of fetal genome recovery methods

\begin{tabular}{|c|c|c|c|}
\hline Category & Current study & Kitzmanet al. & Fan et al. \\
\hline \multicolumn{4}{|c|}{ Parental haplotype inference } \\
\hline $\begin{array}{l}\text { Method for } \\
\text { parental } \\
\text { haplotype } \\
\text { construction }\end{array}$ & $\begin{array}{l}\text { Trio strategy with corresponding grandparents } \\
\text { and CHS }\end{array}$ & $\begin{array}{l}\text { Maternal: fosmid-based approach [26] } \\
\text { Paternal: could not be assessed due to } \\
\text { lowmolecular weight of saliva DNA }\end{array}$ & $\begin{array}{l}\text { Maternal: single-cell approach } \\
\text { [27] } \\
\text { Paternal: not collected }\end{array}$ \\
\hline \multicolumn{4}{|c|}{ Strategy for fetal genome recovery } \\
\hline Paternalallele & $\begin{array}{l}\text { Two different alleles of fetal haplotype, } \\
\text { transmitted from the two parents, were } \\
\text { reconstructed by a HMM model in one step, } \\
\text { including transmitted chromosomes and } \\
\text { recombination breakpoints }\end{array}$ & SBSS & SBSS+ imputation \\
\hline Maternal allele & & $\begin{array}{l}\text { For maternal-only heterozygous sites, they } \\
\text { used AlEto determine whole-block transitions } \\
\text { and HMM to identify assembly errors and } \\
\text { recombination breakpoints. For biparentally } \\
\text { heterozygous sites, maternal alleles were } \\
\text { determined by maternal-only heterozygous } \\
\text { sites within the same block }\end{array}$ & $\begin{array}{l}\text { Allele imbalance estimated by } \\
\text { counting nucleotides specific } \\
\text { to each of the two maternal } \\
\text { alleles }\end{array}$ \\
\hline \multicolumn{4}{|c|}{ Recovery of fetal genome } \\
\hline Genotype & Yes & Yes & Yes \\
\hline Haplotype & Yes & No & No \\
\hline $\begin{array}{l}\text { De-novo } \\
\text { mutation }\end{array}$ & No & Yes & No \\
\hline
\end{tabular}

The dependence on cff-DNA concentration and sequence depth also limits the application of SBSS to early gestation. Therefore, it is an advisable strategy to use the paternal haplotype for noninvasive fetal whole genome recovery, especially for Mendelian disease diagnosis.
Third, the results of the fetal genome recovery were different between the three studies. Kitzman et al. and Fan et al. focused on fetal genotype inference. We tried to recover the fetal haplotype and genotype because the haplotype information is important for complex diseases screening, such as systemic lupus erythematosus [18].

Table 4 Practical performance comparison between fetal genome recovery methods

\begin{tabular}{|c|c|c|c|c|c|c|c|}
\hline \multirow{2}{*}{\multicolumn{2}{|c|}{ Category }} & \multirow[t]{2}{*}{ Current study } & \multicolumn{2}{|c|}{ Kitzman et al. } & \multicolumn{3}{|c|}{ Fan et al. } \\
\hline & & & Trio I1 & Trio G1 & P1T1 & P1T2 & P2T3 \\
\hline \multicolumn{2}{|c|}{ Gestational week } & 13 & 18.5 & 8.14 & 9 & 29 & 39 \\
\hline \multicolumn{2}{|c|}{ Estimated average cff-DNA concentration } & $5.69 \%$ & $13 \%^{\mathrm{a}}$ & $6 \%^{\mathrm{a}}$ & $6 \%^{\mathrm{a}}$ & $16 \%^{\mathrm{a}}$ & $30 \%{ }^{\mathrm{a}}$ \\
\hline \multicolumn{2}{|c|}{ Average sequence depth (fold) } & 43.91 & $78^{\mathrm{a}, \mathrm{b}}$ & $56^{a, b}$ & 52.7 & 20.8 & 10.7 \\
\hline \multicolumn{2}{|c|}{ Fetal gender } & Male & Male & - & Female & Female & Female \\
\hline \multicolumn{8}{|c|}{ Maternal allele } \\
\hline \multicolumn{2}{|c|}{ Predicted rate } & $100 \%$ & $91.4 \%$ & - & $>99.2 \%$ & & \\
\hline \multicolumn{2}{|c|}{ Predictionaccuracy } & $\begin{array}{l}\text { 95.37\% (autosome) } \\
\text { 98.45\% (ChrX) }\end{array}$ & - & - & $>99.8 \%$ & & \\
\hline \multicolumn{8}{|c|}{ Paternal allele } \\
\hline \multicolumn{2}{|c|}{ Predicted rate } & $100 \%$ & - & - & $71.60 \%$ & $72.84 \%$ & $72.94 \%$ \\
\hline \multicolumn{2}{|c|}{ Prediction accuracy } & $98.57 \%$ & - & - & $93.79 \%$ & $95.84 \%$ & $96.56 \%$ \\
\hline \multicolumn{8}{|c|}{ Accuracy of inferred fetal genotype } \\
\hline \multirow[t]{3}{*}{ Autosome } & Paternal-only heterozygous & $99.12 \%, n=65,409$ & $96.8 \%$ & $60.3 \%$ & - & - & - \\
\hline & Maternal-only heterozygous & $95.84 \%, n=66,238$ & $99.3 \%^{c}$ & $95.7 \%^{\mathrm{c}}$ & - & - & - \\
\hline & Biparentally heterozygous & $94.90 \%, n=41,849$ & $98.7 \%^{\mathrm{d}}$ & $91.3 \%^{d}$ & - & - & - \\
\hline ChrX & Maternal-only heterozygous & $98.45 \%, n=1,932$ & - & - & - & - & - \\
\hline
\end{tabular}

- = No data

${ }^{\text {a Approximate. }}$

${ }^{\mathrm{b}}$ Non-duplicate.

${ }^{\mathrm{C}}$ Estimated based on maternal phased sites.

${ }^{d}$ Accuracy of transmitted maternal allele prediction, estimated based on maternal phased sites. 
Besides helping in haplotype-related disease detection, accurate fetal haplotype prediction might be helpful to identify fetal de-novo copy number variations, such as aneuploidy or even microdeletion and microduplication syndromes (Additional file 1, Supplementary Materials).

Fourth, only Kitzman et al. performed a fetal de-novo mutation identification. These mutations were expected to appear within the maternal plasma as rare alleles, like paternal-specific alleles [15]. Ideally, SBSS can identify the fetal de-novo mutations easily. Therefore, Kitzman et al. achieved $88.60 \%$ sensitivity of high-confidence fetal de-novo mutations. However, the systematic error, which was dominated by errors originating during polymerase chain reaction (PCR), introduced hundreds of noisy signals. Even with stringent filters, $>99 \%$ of the candidate sites were false-positive, implying a specificity of approximately $0.84 \%$ in trio I1. Moreover, as mentioned above, the sensitivity of SBSS depends on cffDNA concentration and sequence depth. In our case, only $68.97 \%$ of the high-confidence fetal de-novo mutations could be identified (Additional file 1, Table S4). Hence, effective algorithms are still required for fetal denovo mutation identification.

In conclusion, all three of these methods provide promising solutions for non-invasive fetal genome recovery, with different strengths and weaknesses. The performance of paternal allele recovery indicated the requirement of the paternal haplotype, especially for non-invasive Mendelian disease detection. Therefore, it is wise to choose a suitable approach to obtain the parental haplotype based on the clinical reality, and our data show that a strategy with additional relative samples would be an alternative method. Our strategy of parental haplotype inference provided a practical solution to detect fetal Mendelian diseases non-invasively, especially for couples with a born proband.

Currently, it costs US $\$ 41$ to generate a single gigabyte of sequence data with the Illumina HiSeq 2000 platform [28]; therefore, it would cost at least US\$14,000 to generate the sequence data in this study. With developing technology, the price of the sequencing will drop to US $\$ 1,000$ per genome in the foreseeable future [29]. Consequently, sequence-based approaches will become practical for non-invasive fetal whole genome recovery. Regarding Mendelian diseases, combining our method with exome sequencing technology, it will cost only US $\$ 1,200-1,400$ for each family with a born proband (including 30X exome sequence coverage for the couple, the born proband, and the maternal plasma), which is affordable for many families. Moreover, the developing sequence platforms with shorter turnaround times will significantly broaden the application of sequence-based approaches for fetal genome recovery. For example, the MiSeq platform takes $<48$ h for PE 150 sequencing, meaning pregnant women could receive their results within 1 week. Thus, the advantage of short turnaround times makes us confident that sequence-based approaches for fetal genome recovery will play an increasingly important role in the future.

The development of non-invasive measures for fetal genome recovery will surely bring new insight to prenatal genetic diagnosis. In the case of fetal Mendelian disease identification, sequence-based approaches will provide fast and reliable options to pregnant women, reducing the use of unnecessary invasive procedures. Comprehensive fetal genome sequencing with high accuracy not only enables us to make definitive diagnoses but also provides potential applications in personal medicine, such as identification of allergens [30]. In addition, the easy sampling of sequence-based approaches shows eurytopicity for gestational stage, which may be helpful to make appropriate clinical decisions. For instance, a fetus diagnosed with phenylketonuria in the third trimester would benefit from treatment immediately after delivery [16]. However, the increase in information available to parents will raise ethical questions. For example, in most cases, the influence of a novel fetal mutation is hard to predict. Should a woman be informed if her fetus has a novel mutation of unpredictable consequence? The uncertainty of these mutations may increase the unnecessary anxiety of pregnant women; however, the lack of such information would lead to improper decisions. Thus, the key concern is what kind of information would/should be reported, and this question should be thoroughly discussed within the scientific community and on a societal level.

There are still several limitations of our approach hampering further clinical application. First, the use of commercial microarrays (grandparents or the CHS trios) greatly restricted our study of common SNPs. Therefore, only a small fraction of SNPs were discussed in our study, and we ignored most of the rare variations. In addition, short indels, which could not be located in the parental haplotypes because of the lack of grandparental information, were excluded from our analysis. Short indels not only play an important role in Mendelian disease [31] but also show strong power as markers [32]. At present, target sequences with abundant tag-SNPs are advisable for future studies. Second, our study focused on mutations at the DNA level, which excluded most of the haplotype-associated transgenerational epigenetic modifications [33]. The clinical application of fetal genome recovery will require more robust experimental breakthroughs and algorithms to explore the comprehensiveness of the genome coverage. Third, although we successfully recovered a fetal genome in a case of a singleton pregnancy, accurate genome recovery in cases of twin pregnancy is still unattainable. Currently, the sequence-based approach for non-invasive prenatal diagnosis in twin pregnancies is restricted to 
aneuploidy [34]. The recovery of twins' genomes will greatly broaden the horizon of non-invasive prenatal diagnosis.

\section{Conclusions}

In this study, we introduced an accurate method for fetal genome recovery in one step using maternal plasma sequencing. More than $95 \%$ of the fetal genotypes were inferred successfully, and most importantly, $>95 \%$ of the fetal haplotypes were recovered precisely. As a proof of concept, we propose the clinical application of the recovered genome to non-invasive prenatal diagnosis/screening. In summary, we report an accurate and easy method for non-invasive fetal whole genome recovery by maternal plasma sequencing. An accurate fetal haplotype would enhance the dimensionality of fetal variation detection in prenatal diagnosis/screening and promote the development of fetal medicine. Our results indicate the potential of using sequencing technology in prenatal diagnosis, and they should accelerate the application of sequencing technology in clinical trials.

\section{Additional material}

\section{Additional file 1: Supplementary materials. This file provides a} detailed description of the experimental work and bioinformatics methods of this study (SNP calling, parental haplotype construction, fetal genome recovery). We also performed comprehensive maternal plasma DNA profiling (section 2). This file further contains supplementary Figures S1-5 and Tables S1-3: Figure S1, 'The size distribution of plasma DNA', shows the size distribution of maternal and fetal DNA fragments in DNA. Figure S2, 'The GC content of plasma DNA segments', shows the GC consistency between maternal and paternal DNA segments in plasma. Figure S3, The sequence depth of paternal-specific alleles', shows the sequence depth distribution of paternal-specific alleles in maternal plasma. Figure S4, The distribution of the concentration difference between allele 0 and allele 1 ', shows the concentration difference between the maternal alleles in plasma. Table S1, 'The consistency between SNP calling using NGS data and the Illumina 2.5M array', shows the SNP calling consistency between NGS and the SNP-array. Table S2, The average of estimated concentration of each allele on different chromosomes', shows the average concentration of each allele on different chromosomes calculated by MLE. Table S3, 'The cff-DNA concentration of each chromosome based on paternal-specific alleles', shows the average cff-DNA concentration on each chromosome. Table S4, The read distribution of fetal de-novo mutations', shows the distribution of reads in plasma sequences at high-confidence fetal denovo mutation sites. Table S5, 'The type III errors', lists detailed information of the type III errors of fetal genome recovery.

\section{Abbreviations \\ cff-DNA: cell-free fetal DNA; g-DNA: genomic DNA; HMM: hidden Markov model.}

\section{Authors' contributions}

$X Z, Y L, H Y$, JiW, JuW, SL, and $W W$ managed the project. $Y L, X Z$, and SC designed the analysis principle. SC, XW, XL, XY, CZ, HZ, and FJ performed the data analysis. HG, XP, FC, HJ, PL, LeZ, and LiZ performed the sequencing.
SC, HG, and XP prepared this manuscript. All authors read and approved the final manuscript.

\section{Competing interests}

The authors have read the journal's policy and have the following competing interests to declare: Shengpei Chen, Huijuan Ge, Xuchao Li, Jian Wang, Jun Wang, Huanming Yang, and Xiuqing Zhang have filed patent applications on non-invasive fetal genome recovery by massively parallel sequencing of maternal plasma. The patent number is PCT/CN2012/075478 (date 2012-5-14). The remaining authors declare that they have no competing interests.

\section{Acknowledgements}

The study was funded by Key Laboratory of Cooperation Project in Guangdong Province (2011A060906007), Shenzhen Birth Defect Screening Project Lab (JZF No. [2011] 861), and Key Laboratory Project in Shenzhen (CXB200903110066A and CXB201108250096A). We also sincerely thank our colleagues at BGl for sequencing and Laurie Goodman for writing advises.

\section{Author details}

'BGI-Shenzhen, Shenzhen 518083, China. ${ }^{2}$ State Key Laboratory of Bioelectronics, School of Biological Science and Medical Engineering, Southeast University, Nanjing 210096, China. ${ }^{3}$ School of Bioscience and Bioengineering, South China University of Technology, Guangzhou 510000, China.

Received: 27 August 2012 Revised: 23 January 2013

Accepted: 27 February 2013 Published: 27 February 2013

\section{References}

1. Baird PA: Prenatal screening and the reduction of birth defects in populations. Community Genet 1999, 2:9-17.

2. ACOG Practice Bulletin No. 88, December 2007. Invasive prenatal testing for aneuploidy.. Obstet Gynecol 2007, 110:1459-1467.

3. Tabor A, Philip J, Madsen M, Bang J, Obel EB, Norgaard-Pedersen B: Randomised controlled trial of genetic amniocentesis in 4606 low-risk women. Lancet 1986, 1:1287-1293.

4. Wapner R, Thom E, Simpson JL, Pergament E, Silver R, Filkins K, Platt L, Mahoney M, Johnson A, Hogge WA, Wilson RD, Mohide P, Hershey D, Krantz D, Zachary J, Snijders R, Greene N, Sabbagha R, MacGregor S, Hill L, Gagnon A, Hallahan T, Jackson L: First-trimester screening for trisomies 21 and 18. N Engl J Med 2003, 349:1405-1413.

5. Alfirevic $Z$, Neilson JP: Antenatal screening for Down's syndrome. BMJ 2004, 329:811-812

6. Malone FD, Canick JA, Ball RH, Nyberg DA, Comstock CH, Bukowski R, Berkowitz RL, Gross SJ, Dugoff L, Craigo SD, Timor-Tritsch IE, Carr SR, Wolfe HM, Dukes K, Bianchi DW, Rudnicka AR, Hackshaw AK, LambertMesserlian G, Wald NJ, D'Alton ME: First-trimester or second-trimester screening, or both, for Down's syndrome. N Engl J Med 2005, 353:2001-2011.

7. Herzenberg LA, Bianchi DW, Schroder J, Cann HM, Iverson GM: Fetal cells in the blood of pregnant women: detection and enrichment by fluorescence-activated cell sorting. Proc Natl Acad Sci USA 1979, 76:1453-1455.

8. Bianchi DW, Flint AF, Pizzimenti MF, Knoll JH, Latt SA: Isolation of fetal DNA from nucleated erythrocytes in maternal blood. Proc Natl Acad SCi USA 1990, 87:3279-3283.

9. Cheung MC, Goldberg JD, Kan YW: Prenatal diagnosis of sickle cell anaemia and thalassaemia by analysis of fetal cells in maternal blood. Nat Genet 1996, 14:264-268.

10. Bianchi DW, Williams JM, Sullivan LM, Hanson FW, Klinger KW, Shuber AP: PCR quantitation of fetal cells in maternal blood in normal and aneuploid pregnancies. Am J Hum Genet 1997, 61:822-829.

11. Chiu RW, Chan KC, Gao Y, Lau WY, Zheng W, Leung TY, Foo CH, Xie B, Tsui NB, Lun FM, Zee BC, Lau TK, Cantor CR, Lo YM: Noninvasive prenatal diagnosis of fetal chromosomal aneuploidy by massively parallel genomic sequencing of DNA in maternal plasma. Proc Natl Acad Sci USA 2008, 105:20458-20463.

12. Tong YK, Ding C, Chiu RW, Gerovassili A, Chim SS, Leung TY, Leung TN, Lau TK, Nicolaides KH, Lo YM: Noninvasive prenatal detection of fetal 
trisomy 18 by epigenetic allelic ratio analysis in maternal plasma: Theoretical and empirical considerations. Clin Chem 2006, 52:2194-2202.

13. Fan HC, Blumenfeld YJ, Chitkara U, Hudgins L, Quake SR: Noninvasive diagnosis of fetal aneuploidy by shotgun sequencing DNA from maternal blood. Proc Natl Acad Sci USA 2008, 105:16266-16271.

14. Lo YM, Chan KC, Sun $H$, Chen EZ, Jiang P, Lun FM, Zheng YW, Leung TY, Lau TK, Cantor CR, Chiu RW: Maternal plasma DNA sequencing reveals the genome-wide genetic and mutational profile of the fetus. Sci Transi Med 2010, 2:61ra91.

15. Kitzman JO, Snyder MW, Ventura M, Lewis AP, Qiu R, Simmons LE, Gammill HS, Rubens CE, Santillan DA, Murray JC, Tabor HK, Bamshad MJ, Eichler EE, Shendure J: Noninvasive whole-genome sequencing of a human fetus. Sci Transl Med 2012, 4:137ra176.

16. Fan HC, Gu W, Wang J, Blumenfeld YJ, El-Sayed YY, Quake SR: Non-invasive prenatal measurement of the fetal genome. Nature 2012, 487:320-324.

17. Lo YM, Tein MS, Lau TK, Haines CJ, Leung TN, Poon PM, Wainscoat JS, Johnson PJ, Chang AM, Hjelm NM: Quantitative analysis of fetal DNA in maternal plasma and serum: implications for noninvasive prenatal diagnosis. Am J Hum Genet 1998, 62:768-775.

18. Namjou B, Sestak AL, Armstrong DL, Zidovetzki R, Kelly JA, Jacob N, Ciobanu V, Kaufman KM, Ojwang JO, Ziegler J, Quismorio FP Jr, Reiff A, Myones BL, Guthridge JM, Nath SK, Bruner GR, Mehrian-Shai R, Silverman E, Klein-Gitelman M, McCurdy D, Wagner-Weiner L, Nocton JJ, Putterman C, Bae SC, Kim YJ, Petri M, Reveille JD, Vyse TJ, Gilkeson GS, Kamen DL, et al: High-density genotyping of STAT4 reveals multiple haplotypic associations with systemic lupus erythematosus in different racial groups. Arthritis Rheum 2009, 60:1085-1095.

19. Levy S, Sutton G, Ng PC, Feuk L, Halpern AL, Walenz BP, Axelrod N, Huang J, Kirkness EF, Denisov G, Lin Y, MacDonald JR, Pang AW, Shago M, Stockwell TB, Tsiamouri A, Bafna V, Bansal V, Kravitz SA, Busam DA, Beeson KY, McIntosh TC, Remington KA, Abril JF, Gill J, Borman J, Rogers YH, Frazier ME, Scherer SW, Strausberg RL, et al: The diploid genome sequence of an individual human. PLOS Biol 2007, 5:e254.

20. Wang J, Wang W, Li R, Li Y, Tian G, Goodman L, Fan W, Zhang J, Li J, Guo Y, Feng B, Li H, Lu Y, Fang X, Liang H, Du Z, Li D, Zhao Y, Hu Y, Yang Z, Zheng H, Hellmann I, Inouye M, Pool J, Yi X, Zhao J, Duan J, Zhou Y, Qin J, Ma L, et al: The diploid genome sequence of an Asian individual. Nature 2008, 456:60-65.

21. Browning BL, Browning SR: A unified approach to genotype imputation and haplotype-phase inference for large data sets of trios and unrelated individuals. Am J Hum Genet 2009, 84:210-223.

22. OMIM.. [http://www.omim.org].

23. Hindorff $L A$, Sethupathy $P$, Junkins $H A$, Ramos EM, Mehta JP, Collins FS, Manolio TA: Potential etiologic and functional implications of genomewide association loci for human diseases and traits. Proc Natl Acad SCi USA 2009, 106:9362-9367.

24. Miura K, Yoshiura K, Miura S, Shimada T, Yamasaki K, Yoshida A, Nakayama D, Shibata Y, Niikawa N, Masuzaki H: A strong association between human earwax-type and apocrine colostrum secretion from the mammary gland. Hum Genet 2007, 121:631-633.

25. Yoshiura K, Kinoshita A, Ishida T, Ninokata A, Ishikawa T, Kaname T, Bannai M, Tokunaga K, Sonoda S, Komaki R, Ihara M, Saenko VA, Alipov GK, Sekine I, Komatsu K, Takahashi H, Nakashima M, Sosonkina N, Mapendano CK, Ghadami M, Nomura M, Liang DS, Miwa N, Kim DK Garidkhuu A, Natsume N, Ohta T, Tomita H, Kaneko A, Kikuchi M, et al: A SNP in the ABCC11 gene is the determinant of human earwax type. Nat Genet 2006, 38:324-330

26. Kitzman JO, Mackenzie AP, Adey A, Hiatt JB, Patwardhan RP, Sudmant PH, $\mathrm{Ng} \mathrm{SB}$, Alkan C, Qiu R, Eichler EE, Shendure J: Haplotype-resolved genome sequencing of a Gujarati Indian individual. Nat Biotechnol 2011, 29:59-63.

27. Fan HC, Wang J, Potanina A, Quake SR: Whole-genome molecular haplotyping of single cells. Nat Biotechnol 2011, 29:51-57.

28. Quail MA, Smith M, Coupland P, Otto TD, Harris SR, Connor TR, Bertoni A, Swerdlow HP, Gu Y: A tale of three next generation sequencing platforms: comparison of lon Torrent, Pacific Biosciences and Illumina MiSeq sequencers. BMC Genomics 2012, 13:341.

29. Defrancesco L: Life Technologies promises $\$ 1,000$ genome. Nat Biotechnol 2012, 30:126.

30. Zhang Y, Xi L, Zhao YM, Zhao LP, Zhang L: [Association between singlenucleotide polymorphisms in the IRAK-4 gene and allergic rhinitis]. Zhonghua Er Bi Yan Hou Tou Jing Wai Ke Za Zhi 2012, 47:476-480.
31. Ng SB, Buckingham K, Lee C, Bigham AW, Tabor HK, Dent KM, Huff CD, Shannon PT, Jabs EW, Nickerson DA, Shendure J, Bamshad MJ: Exome sequencing identifies the cause of a mendelian disorder. Nat Genet 2010, 42:30-35.

32. Vali U, Brandstrom M, Johansson M, Ellegren H: Insertion-deletion polymorphisms (indels) as genetic markers in natural populations. $B M C$ Genet 2008, 9:8.

33. Youngson NA, Whitelaw E: Transgenerational epigenetic effects. Annu Rev Genomics Hum Genet 2008, 9:233-257.

34. Canick JA, Kloza EM, Lambert-Messerlian GM, Haddow JE, Ehrich M, van den Boom D, Bombard AT, Deciu C, Palomaki GE: DNA sequencing of maternal plasma to identify Down syndrome and other trisomies in multiple gestations. Prenat Diagn 2012, 1-5.

doi:10.1186/gm422

Cite this article as: Chen et al:: Haplotype-assisted accurate non-invasive fetal whole genome recovery through maternal plasma sequencing. Genome Medicine 2013 5:18.

\section{Submit your next manuscript to BioMed Central and take full advantage of:}

- Convenient online submission

- Thorough peer review

- No space constraints or color figure charges

- Immediate publication on acceptance

- Inclusion in PubMed, CAS, Scopus and Google Scholar

- Research which is freely available for redistribution

Submit your manuscript at www.biomedcentral.com/submit
Biomed Central 